\title{
Analysis of MC-CDMA System in Mobile Communications
}

\author{
M. F. Ghanim \\ Computer Engineering Department, College of Engineering, University of Mosul, Mosul, Iraq \\ Email: mayada_faris@yahoo.com
}

M. F. L. Abdullah

Faculty of Electrical and Electronic Engineering, University Tun Hussein onn Malaysia, Johor, Malaysia Email: faiz@uthm.edu.my

\begin{abstract}
Wireless communication plays an important role in our daily life. One of the most important techniques which is Candidate for the fourth generation is Multicarrier Code Division Multiple Access (MCCDMA) due to its high data rate. This research paper presents the MC-CDMA system using different modulation techniques. The variety of modulation types are depended in order to show the effects of modulation index and type on broadcasting data. The bit error rate of the system is plotted for a range of signal to noise ratio so that the effect of modulation on the MC-CDMA system will be evident. Actually, the simulation results show that QAM gives less bit error rate that makes MCCDMA more flexible and suitable for mobile communication next generation technology. Also, the peak-to-average power (PAPR) of MC-CDMA is analyzed to show that high PAPR is the main disadvantage of MC-CDMA system then the possible solutions for this problem are discussed in this research paper.
\end{abstract}

Index Terms - MC-CDMA, PAPR, Spreading Codes, QAM, PSK, Modulation

\section{Introduction}

High data rate digital communications is extremely demanded nowadays. This demand has driven research in recent years with orthogonal frequency division multiplexing (OFDM) emerging as one of the most promising technologies. OFDM uses dense subcarrier stacking, due to` orthogonality, making it a spectrally efficient modulation technique with robustness in frequency selective fading channels [1].

Multi-carrier code division multiple access (MCCDMA) combines OFDM with code division multiple access (CDMA) offering gains in both frequency and code diversity and provides OFDM systems with a multiple access mechanism.
MC-CDMA may be implemented by applying the CDMA spreading in either the frequency domain and/or the time domain, with each having its own advantages and disadvantages [1].

The family of multicarrier code division multiple access (MC-CDMA) is an advanced broadband wireless transmission technology combining orthogonal frequency-division multiplexing (OFDM) and spread spectrum techniques. Thanks to this combination, the MC-CDMA family can inherit several competitive properties, e.g., robustness against the frequency selective fading channel, flexibility in system design, low detection complexity, and lower peak-to-average power ratio. In recent years, many aspects have been investigated for the conventional MC-CDMA systems, including the maintenance of quality of service, elimination of interference, as well as multiple access interference (MAI), management of spreading code resources, and the design of advanced detection schemes [2].

The remainder of this paper is organized as follows: Section 2 focuses on all the details of MC-CDMA transmitter and receiver. Section 3 describes WalshHadamard codes that may are used with MC-CDMA system in this work. One of the most important parameters of MC-CDMA which takes the attention of researchers nowadays is PAPR. This property is discussed with Peak Power reduction techniques of A Multi-Carrier CDMA Signal in section 4 and 5. The proposed system with its simulation and the discussion of results are listed in details in section 6. Finally, the main conclusions from this research paper are in section 7.

\section{Principles of Multicarrier CDMA}

In MC-CDMA, instead of applying spreading sequences in the time domain, we can apply them in the frequency domain, mapping a different chip of a spreading sequence to an individual OFDM subcarrier. Hence each OFDM subcarrier has a data rate identical 
to the original input data rate and the multicarrier system "absorbs" the increased rate due to spreading in a wider frequency band [3]. The transmitted signal of the ith data symbol of the $\mathrm{j}^{\text {th }}$ user $\mathrm{s}_{\mathrm{i}}^{\mathrm{j}}(\mathrm{t})$ is written as:

$$
s_{i}^{j}(t)=\sum_{k=0}^{N-1} b_{i}^{j} c_{k}^{j} e^{2 \pi\left(f_{0}+k f_{d}\right) t} p(t-i T),
$$

where

- $\mathrm{N}$ is the number of subcarriers

- $b_{i}{ }^{j}$ is the $i^{\text {th }}$ message symbol of the jth user

- $\mathrm{c}_{\mathrm{k}}{ }^{\mathrm{j}}$ represents the $\mathrm{k}^{\text {th }}$ chip, $\mathrm{k}=0, \ldots, \mathrm{N}-1$, of the spreading sequence of the jth user

- $\mathrm{f}_{0}$ is the lowest subcarrier frequency

- $f_{d}$ is the subcarrier separation

- $\mathrm{p}(\mathrm{t})$ is a rectangular signalling pulse shifted in time given by:

$$
p(t) \triangleq \begin{cases}1 & \text { for } 0 \leq t \leq T \\ 0 & \text { otherwise }\end{cases}
$$

$$
\frac{1}{T} \text { bps } \quad \frac{N}{T} \text { cps } \quad \frac{1}{T} \text { sps }
$$

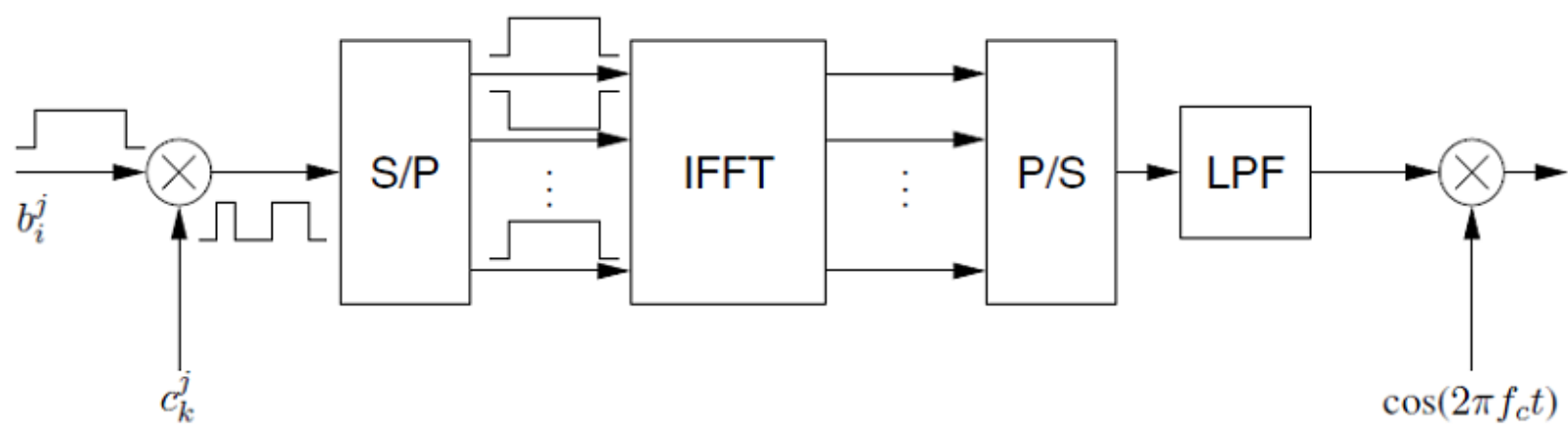

\section{Spreader OFDM Modulator}

Fig. 1: Transmitter schematic of MC-CDMA

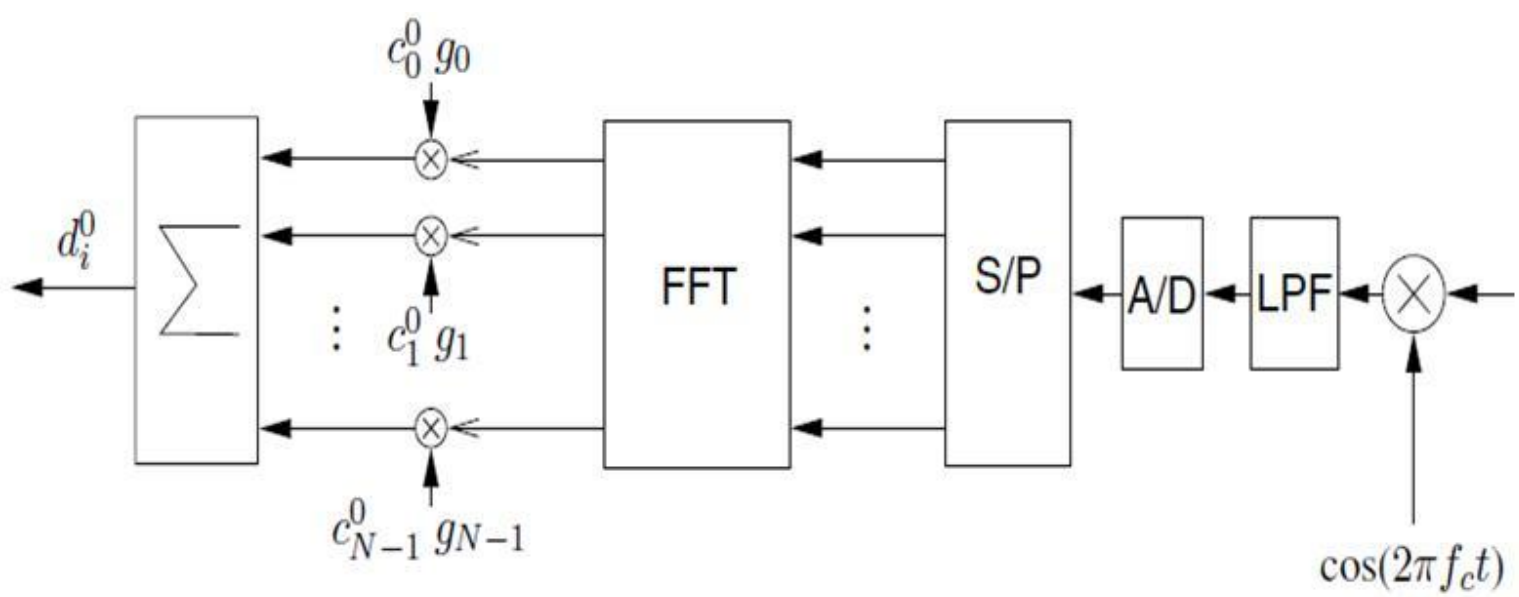

Fig. 2: Receiver schematic of MC-CDMA 
At the MC-CDMA receiver shown in Fig. 2 each carrier's symbol, i.e. the corresponding chip $c_{k}{ }^{j}$ of user $j$, is recovered using FFT after sampling at a rate of N/T samples/sec and the recovered chip sequence is correlated with the desired user's spreading code in order to recover the original information, $b_{i}{ }^{j}$. Let us define the ith received symbol at the $\mathrm{k}^{\text {th }}$ carrier in the downlink as [3]:

$$
r_{k, i}=\sum_{j=0}^{J-1} H_{k} b_{i}^{j} c_{k}^{j}+n_{k, i}
$$

where $\mathrm{J}$ is the number of users, $\mathrm{H}_{\mathrm{k}}$ is the frequency response of the $\mathrm{k}^{\text {th }}$ subcarrier and $\mathrm{n}_{\mathrm{k}, \mathrm{I}}$ is the corresponding noise sample. The MC-CDMA receiver of the 0-th user multiplies $r_{k, i}$ of Equation 3 by its spreading sequence chip, $\mathrm{c}_{\mathrm{k}}^{0}$, as well as by the gain, $\mathrm{g}_{\mathrm{k}}$, which is given by the reciprocal of the estimated channel transfer factor of subcarrier $\mathrm{k}$, for each received subcarrier symbol for $\mathrm{k}=0, \cdot, \mathrm{N}-1$, and sums all these products, in order to arrive at the decision variable, $\mathrm{d}^{0}{ }_{\mathrm{i}}$, which is given by:

$$
d_{i}^{0}=\sum_{k=0}^{N-1} c_{k}^{0} g_{k} r_{k, i}
$$

Without the frequency domain equalization of the received subcarrier sy mbols, the orthogonality between the different users cannot be maintained [3].

\section{Walsh-hadamard Codes}

Various spreading codes exist which can be distinguished with respect to orthogonality, correlation properties, implementation complexity and peak-toaverage power ratio (PAPR). The selection of the spreading code depends on the scenario. In the synchronous downlink, orthogonal spreading codes are of advantage, since they reduce the multiple access interference compared to non-orthogonal sequences. [4].

Orthogonal Walsh-Hadamard codes are simple to generate recursively by using the following Hadamard matrix generation,

$$
\mathbf{C}_{L}=\left[\begin{array}{rr}
\mathbf{C}_{L / 2} & \mathbf{C}_{L / 2} \\
\mathbf{C}_{L / 2} & -\mathbf{C}_{L / 2}
\end{array}\right], \quad \forall L=2^{m}, \quad m \geq 1, \quad \mathbf{C}_{1}=1 .
$$

The maximum number of available orthogonal spreading codes is $\mathrm{L}$ which determines the maximum number of active users $\mathrm{K}$.

The Hadamard matrix generation can also be used to perform an L-ary Walsh-Hadamard modulation which in combination with PN spreading can be applied in the uplink of MC-CDMA systems.

\section{Specifications of Peak Power of MC-CDMA Signal}

The variation of the envelope of a mu lti-carrier signal can be defined by the peak-to average power ratio (PAPR) which is given by

$$
P A P R=\frac{\max \left|x_{v}\right|^{2}}{\frac{1}{N_{c}} \sum_{v=0}^{N_{c}-1}\left|x_{v}\right|^{2}}
$$

The values $\mathrm{xv}, \mathrm{v}=0, \ldots, \mathrm{Nc}-1$, are the time samples of an OFDM symbol. An additional measure to determine the envelope variation is the crest factor (CF) which is

$$
C F=\sqrt{P A P R}
$$

By appropriately selecting the spreading code, it is possible to reduce the PAPR of the multi-carrier signal. This PAPR reduction can be of advantage in the uplink where low power consumption is required in the terminal station.

The uplink signal assigned to userk results in

$$
x_{v}=x_{v}^{(k)}
$$

The PAPR for different spreading codes can be upper-bounded for the uplink by assuming that $\mathrm{Nc}=\mathrm{L}$. The PAPR bounds for Walsh-Hadamard is less than or equal double L. The PAPR bound for Golay codes and Zadoff-Chu codes is independent of the spreading code length. When $\mathrm{Nc}$ is a multiple of $\mathrm{L}$, the PAPR of the Walsh-Hadamard code is upper-bounded by $2 \mathrm{Nc}$.

$$
P A P R \leq \frac{2 \max \left\{\left|\sum_{l=0}^{L-1} c_{l}^{(k)} e^{j 2 \pi l t / T_{s}}\right|^{2}\right\}}{L}
$$

The time samples of a down link multi-carrier symbol assuming synchronous transmission are given as

$$
x_{v}=\sum_{k=0}^{K-1} x_{v}^{(k)}
$$

The PAPR of an MC-CDMA down link signal with $\mathrm{K}$ users and $\mathrm{Nc}=\mathrm{L}$ can be upper bounded By [4]

$$
P A P R \leq \frac{2 \max \left\{\sum_{k=0}^{K-1}\left|\sum_{l=0}^{L-1} c_{l}^{(k)} e^{j 2 \pi t / T_{s}}\right|^{2}\right\}}{L}
$$




\section{Peak to Average Power Ratio Reduction Techniques}

Many techniques have been studied for reducing PAPR of a transmitted signal. As known MC-CDMA is a co mbination between CDMA and OFDM. Techniques for PAPR reduction of OFDM and MC-CDMA signals are the same and can be broadly categorized into three main concepts [5]:

1. Clipping and filtering: The time-domain signal is clipped to a predefined level. This causes spectral leakage into adjacent channels, resulting in reduced spectral efficiency as well as in-band noise degrading the bit error rate performance. Out-of-band radiation caused by the clipping process can, however, be reduced by filtering.

If discrete signals are clipped directly, the resulting clipping noise will all fall in band and thus cannot be reduced by filtering. To avoid this problem, one solution consists of oversampling the original signal by padding the input signal with zeros and processing it using a longer IFFT. The oversampled signal is clipped and then filtered to reduce the out-of-band radiation.

2. Selected mapping: Multiple transmit signals which represent the same OFDM data symbol are generated by multiplying the OFDM symbol by different phase vectors. The representation with the lowest PAPR is selected. To recover the phase information, it is of course necessary to use separate control signaling to indicate to the receiver which phase vector was used.

3. Coding techniques: These techniques consist of finding the code words with the lowest PAPR from a set of codewords to map the input data. A look-up table may be used if $\mathrm{N}$ is small. It is shown that complementary codes have good properties to combine both PAPR and forward error correction.
The latter two concepts are not applicable in the context of LTE; selected mapping would require additional signaling, while techniques based on codeword selection are not compatible with the data scrambling used in the LTE downlink.

\section{System Model and Results}

The MC-CDMA proposed system is simulated using Matlab environment. The system was simulated over Rayleigh Fading and Additive White Gaussian Noise (AWGN) channel model to show the effect of its bit error rate relating to signal to noise ratio.

MC-CDMA system is simulated using Walsh code and this represents an advantage because orthogonal codes have a cross-correlation equal to zero; i.e., they do not interfere with each other.

One of the most important parameters in the MCCDMA system is the sampling frequency. Value of $20 \mathrm{MHz}$ is depended with size of FFT equals to 64 subcarriers. As a result the data symbol duration will be $3.2 \mu \mathrm{s}$. On the other side the number of cyclic prefix $(\mathrm{CP})$ is 16 with a cyclic prefix duration equals to $0.8 \mu \mathrm{s}$.

At these conditions the system will have total subcarriers equals to 80 .

Phase shift keying, PSK, is widely used these days within a whole communications systems. It is particularly well suited to the growing area of data communications. PSK, phase shift keying enables data to be carried on a radio communications signal in a more efficient manner some other forms of modulation.

Fig. 3 shows the MC-CDMA system implemented firstly with 32 PSK. Each symbol needs 5 bits to be presented and the symbol rate will be $1 / 5$ of bit rate.

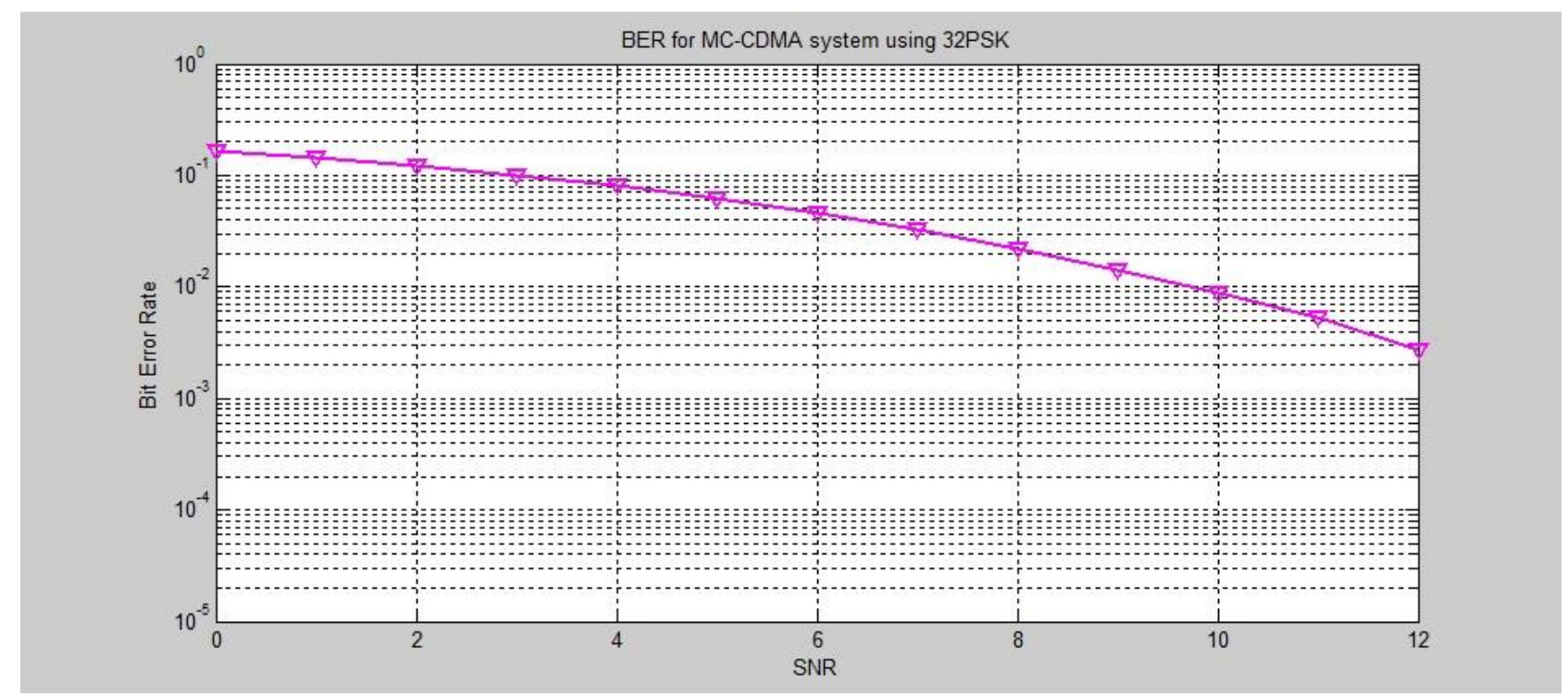

Fig. 3: BER for MC-CDMA system using 32 PSK modulation technique 
When the modulation index is reduced the bit error rate improved and the system became more reliable than before as shown in Fig. 4. In general the higher order forms of modulation allow higher data rates to be carried within a given bandwidth but at the same time BER will be increased.

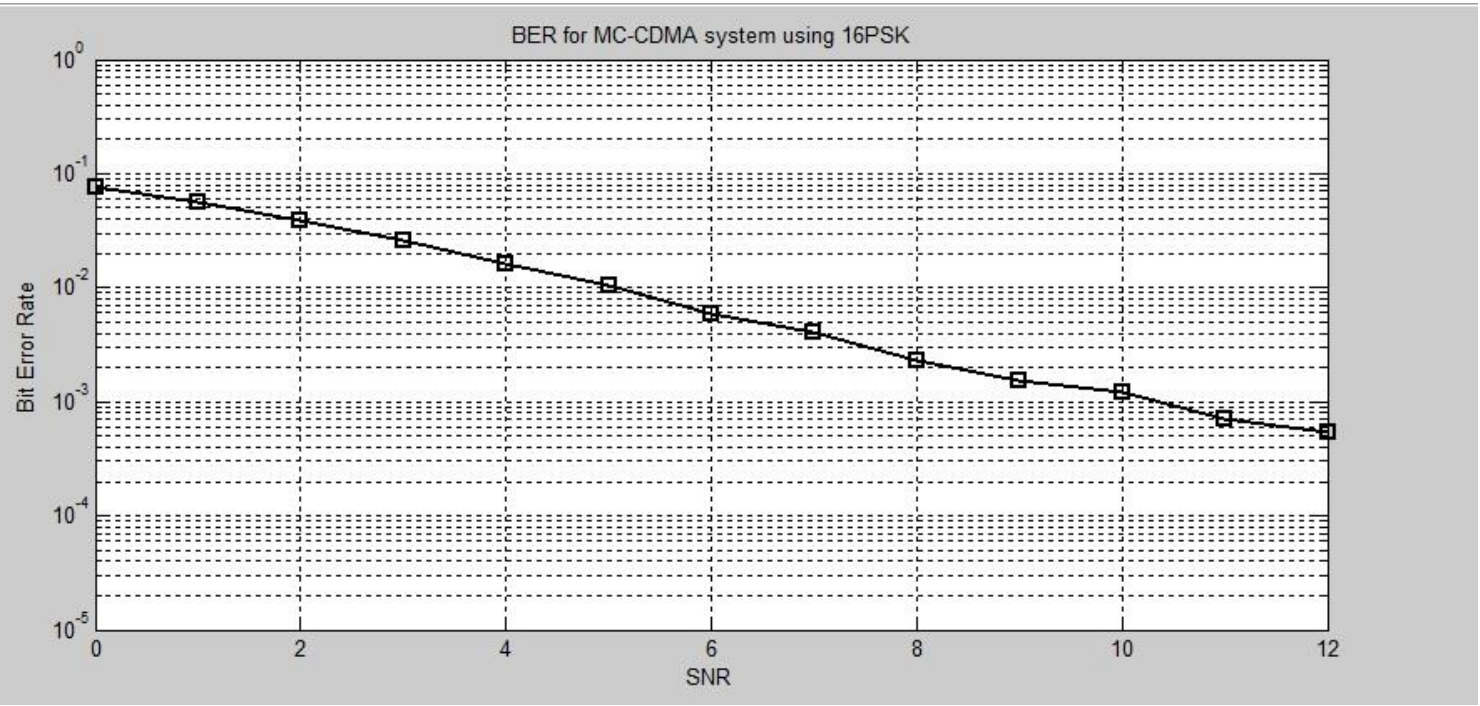

Fig. 4: BER for MC-CDMA system using 16 PSK modulation technique

8PSK, or Eight Phase-Shift Keying, uses eight distinct phase-shifts. These occur at 0 degrees, 45 degrees, 90 degrees, 135 degrees, 180 degrees, 225 degrees, 270 degrees and 315 degrees. Each phase can be expressed by a 3-bit number ( 2 to the power of 3 is equal to 8). Therefore, 8PSK transmits 3-bit symbols per cycle.

Fig. 5 shows how the BER is reduced with 8PSK which makes MC-CDMA more reliable and trusted in mobile communications.

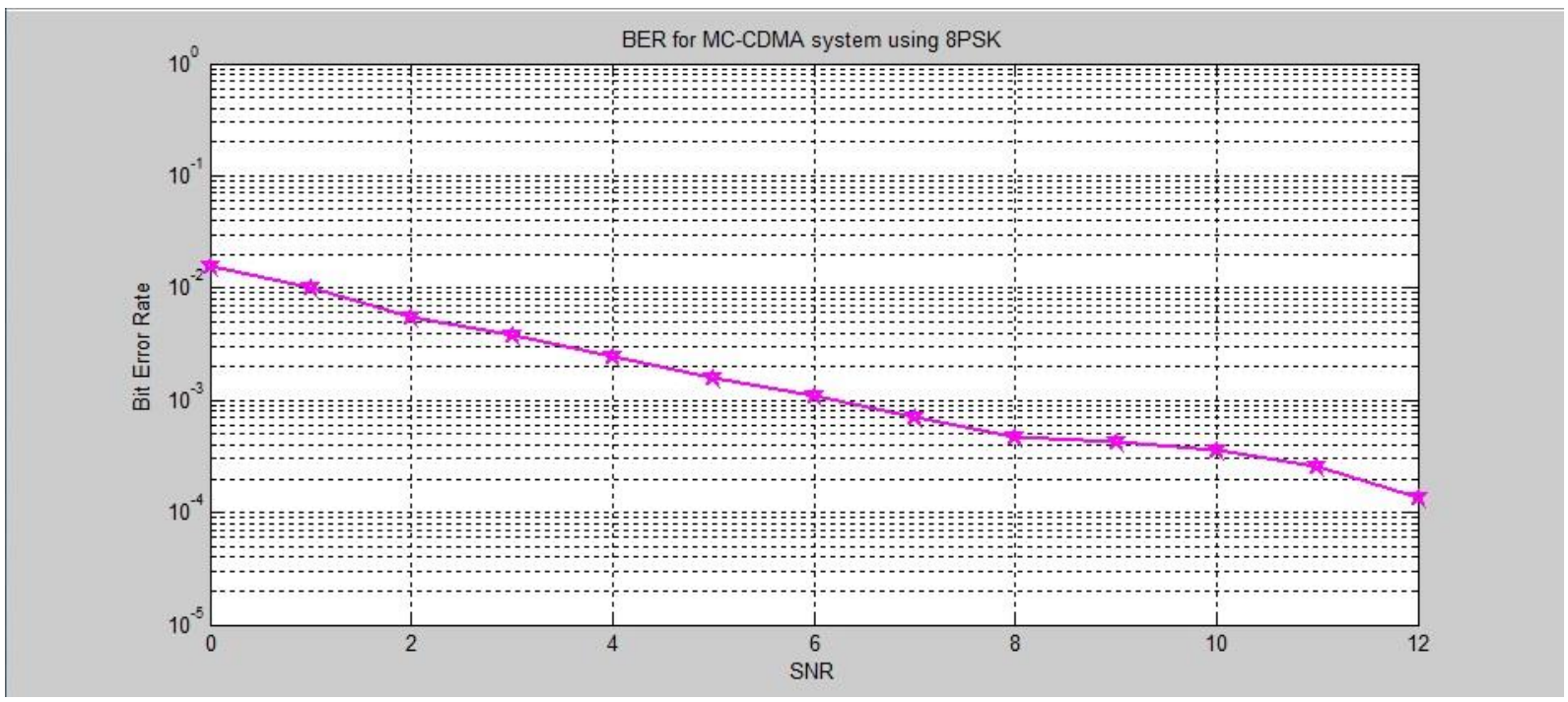

Fig. 5: BER for MC-CDMA system using 8 PSK modulation technique

Quadrature Phase-Shift Key ing QPSK or 8PSK, uses four distinct phase-shifts to encode data. These phaseshifts are 45 degrees, 135 degrees, 225 degrees and 315 degrees. Fig. 6 shows the MC-CDMA system using 4PSK modulation technique. The BER is reduced compared to $8 \mathrm{PSK}$ but $8 \mathrm{PSK}$ trans mits more bits per cycle when compared to QPSK, so 8PSK can achieve a higher data rate at the same frequency than 4PSK. For example, at a carrier-wave frequency of 1000 symbols per second, QPSK trans mits 2000 bits, while 8PSK transmits 3000 bits. 


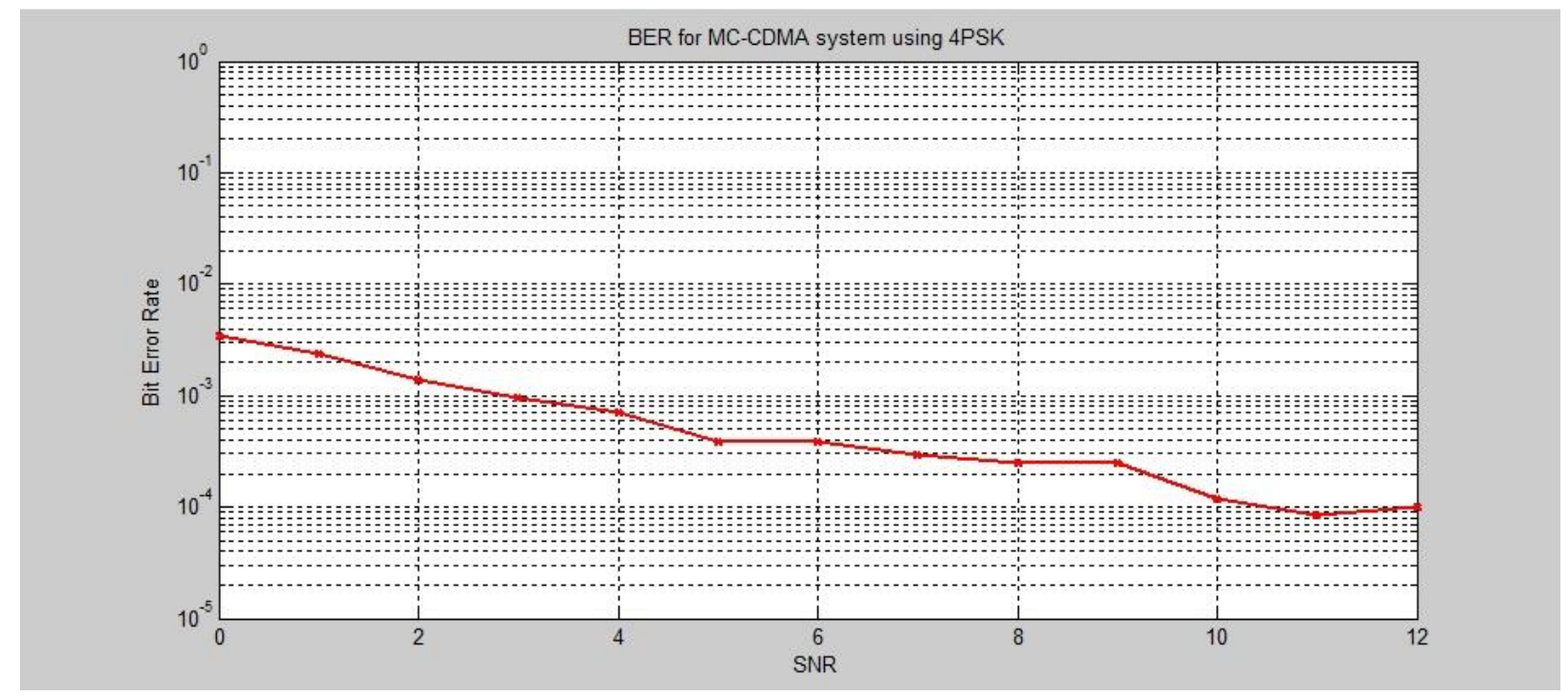

Fig. 6: BER for MC-CDMA system using QPSK modulation technique

When BPSK is used ,the signal is special. It lies in one axis, $\mathrm{x}$-axis. It has no $\mathrm{y}$-axis projection. The vector flip-flops on the $\mathrm{x}$-axis depending on the value of the bit. This case reduces the BER as shown in Fig. 7.

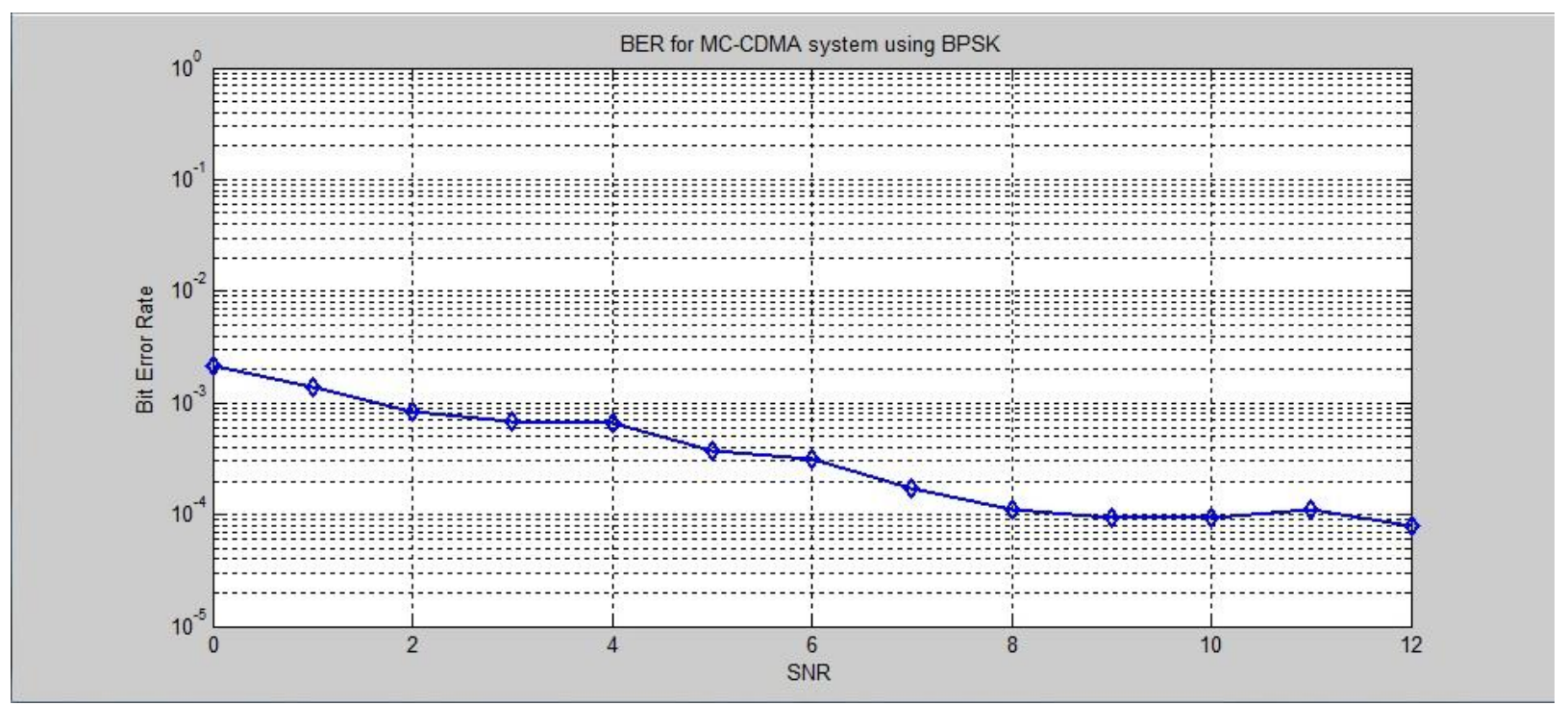

Fig. 7: BER for MC-CDMA system using BPSK modulation technique

The MC-CDMA system with PSK modulation technique is power effective and the modulation is the simplest compared to other techniques, but when QAM is used in MC $=$ CDMA systems the BER is reduced and QAM appears to increase the efficiency of transmission for radio communications systems because of utilizing both amplitude and phase variations. In fact Quadrature PSK (QPSK) is like 4QAM without amplitude modulation. QPSK uses four phase angles to represent each two bits of input; however, the amplitude remains constant.

Fig. 8 shows the MC-CDMA system with QAM modulation technique which gives the best results and minimum bit error rate at different values of signal to noise ratio. 


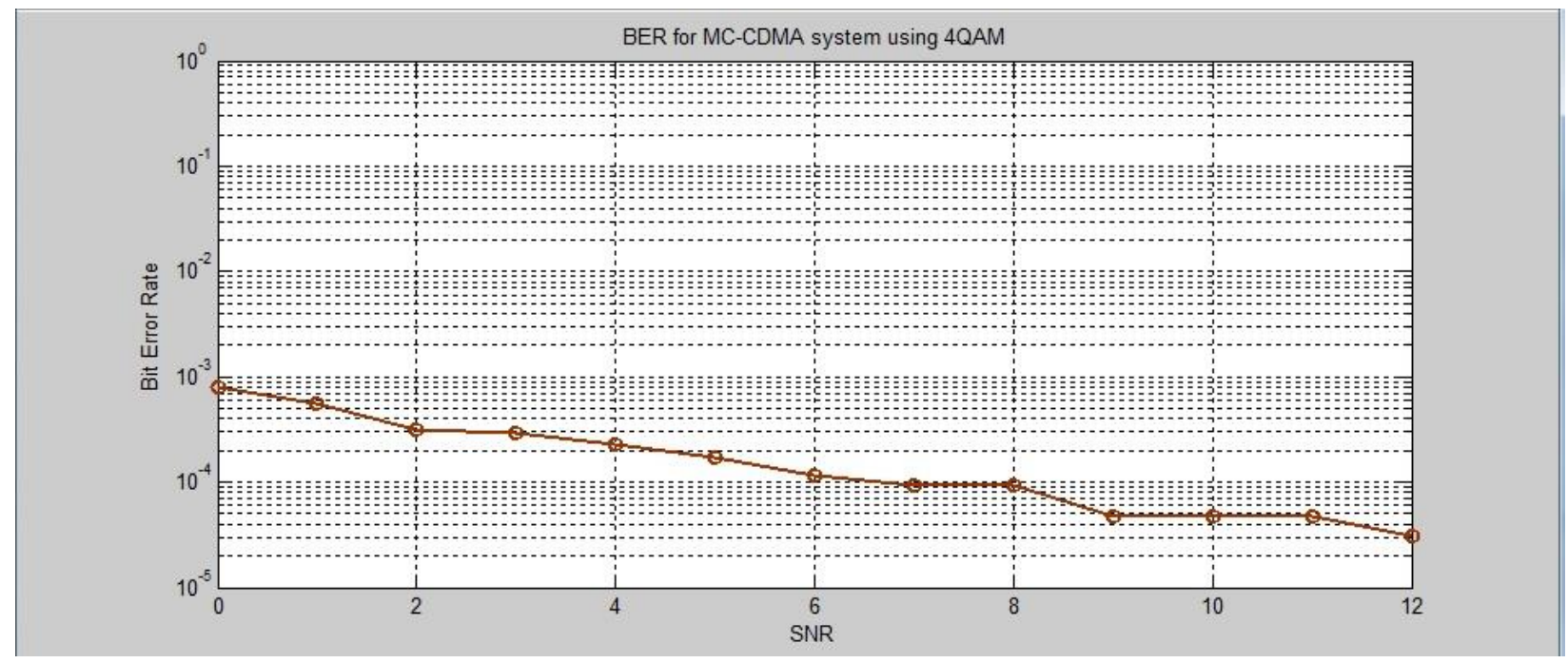

Fig. 8: BER for MC-CDMA system using QAM modulation technique

Finally, PAPR is a performance measurement that is indicative of the power efficiency of the transmitter. In case of an ideal linear power amplifier where linear amplification is achieved up to the saturation point, the maximum power is reached efficiency when the amplifier is operating at the saturation point. A positive PAPR in $\mathrm{dB}$ means that power backoff is needed to operate in the linear region of the power amplifier and high PAPR degrades the transmit power efficiency performance [6].

As shown in Fig. 9, MC-CDMA has high value of PAPR Convergent to 10. Actually this is the main disadvantage of MC-CDMA system in mobile communications.

Nowadays the researchers are focusing on how to reduce PAPR in different methods, while there are main four schemes to reduce PAPR in MC-CDMA system: clipping \& peak windowing, block coding, selected mapping (SLM) and partial transmit sequences (PTS) scheme. It is known that PTS can effectively reduce the PAPR by increasing the number of sub-blocks without modifying the number of subcarriers [7].

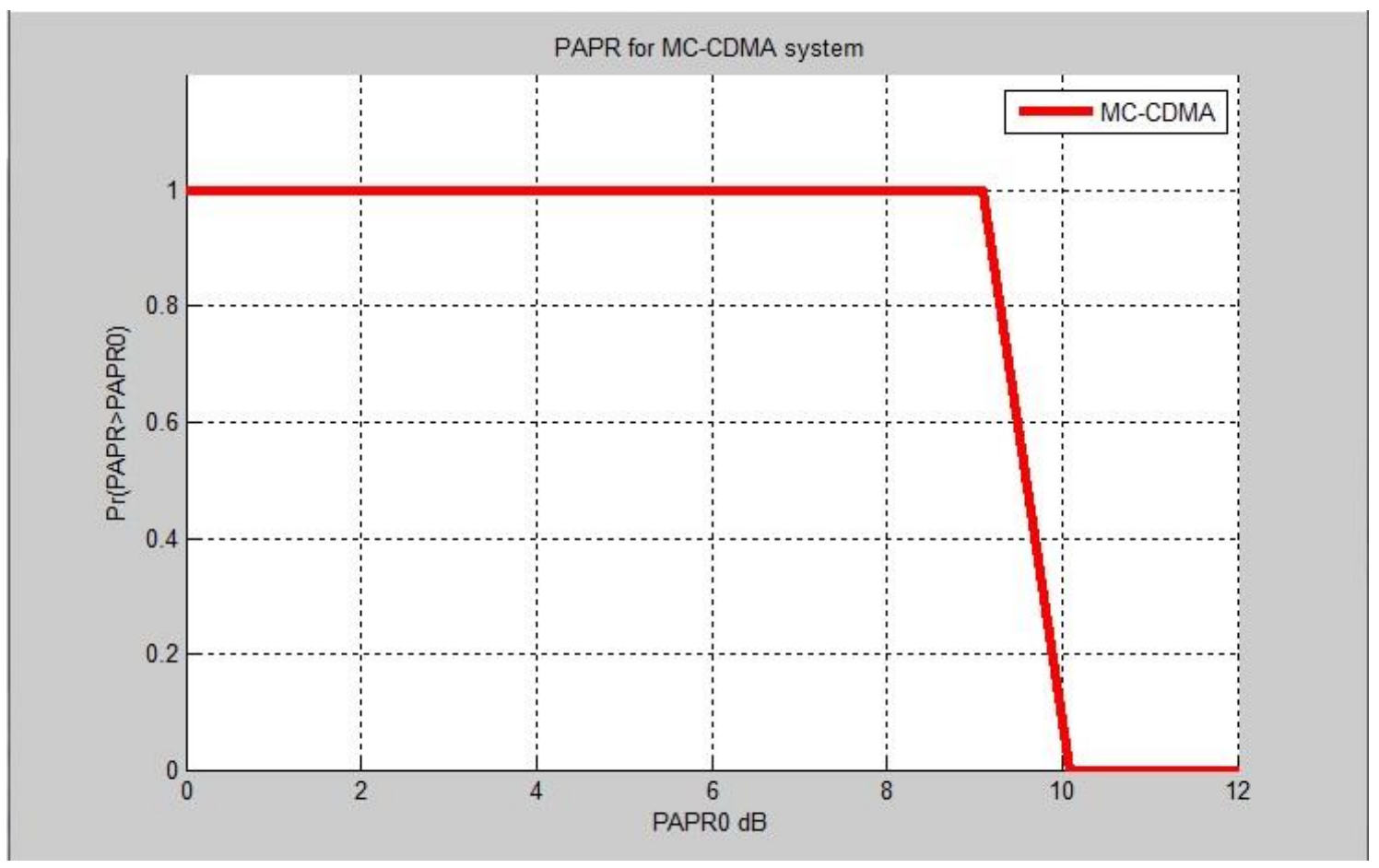

Fig. 9: PAPR of MC-CDMA system 


\section{Conclusions}

In this paper, MC-CDMA performance and analysis are tested to investigate the fact of low bit error rate of MC-CDMA system. The system was simulated by computer for different parameters and using Walsh code which represents an advantage because orthogonal codes have a cross-correlation equal to zero; i.e., they do not interfere with each other. The data that will be broadcasted over the channel is modulated through various modulation techniques. It is noticeable that the bit error rate is converged for different types of modulation techniques but QAM gives the best results and minimu $\mathrm{m}$ bit error rate at different values of signal to noise ratio. On the other hand, PSK is the most popular technique in wireless systems due to its simplicity and suitable cost. Finally, MC-CDMA has high value of PAPR Convergent to 10 and this represents the main disadvantage of MC-CDMA system in mobile communications.

\section{References}

[1] N. d. Figueiredo, L. P. Linde, "MC-CDMA with Blind Channel Estimation and Adaptive Reception," Southern Africa Telecommunication Networks and Applications Conference (SATNAC) , Sep 2011, pp.224-226.

[2] C. Chang, "An Interference-Avoidance Code Assignment Strategy for the Hierarchical TwoDimensional-Spread MC-DS-CDMA System: A Prototype of Cognitive Radio Femtocell System," IEEE Transactions on Vehicular Technology, vol. 61, no. 1, Jan2012, pp.166-167.

[3] L. Hanzo, T. Keller, " OFDM and MC-CDMA A Primer ," John Wiley \& Sons Ltd, England, 2006, pp.211-214.

[4] K. Fazel, S. Kaiser, "Multi-Carrier and Spread Spectrum Systems," John Wiley \& Sons Ltd,2003,pp.52-55.

[5] S. Sesia, I. Toufik, M. Baker, "LTE - The UMTS Long Term Evolution from Theory to Practice," John Wiley \& Sons Ltd, 2009, pp. 122-123.

[6] M. Salah, G. Abdel-Fadeel and Z. B. Nossair, " Peak to Average Power Ratio Reduction in Single Carrier OFDMA Systems," 13th International Conference on Aerospace Sciences \& Aviation Technology, ASAT- 13, Egypt, pp.5, May 2009.

[7] Y. Zhang, Q. Ni1, H. Chen, and Y. Song, "An Intelligent Genetic Algorithm for PAPR reduction in a Multi-Carrier CDMA Wireless System," Wireless Communications and Mobile Computing Conference (IWCMC '08), 1052-1057, 2008.

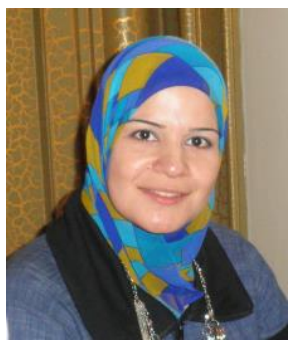

Mayada Faris Ghanim graduated from Computer Engineering Department / College of Engineering at University of Mosul in 2004 and completed her Master in Computer Engineering at the same college in 2007. Currently she is studying doctorate of Electrical and Electronics Engineering at University Tun Hussein Onn Malaysia (UTHM) from 2010. Since 2006 she is working as engineer at the University of the Mosul-Iraq. She has published many refereed journal and conference papers. She is a member of IAENG, SIE, SDIWC, IACSIT and SCIEI.

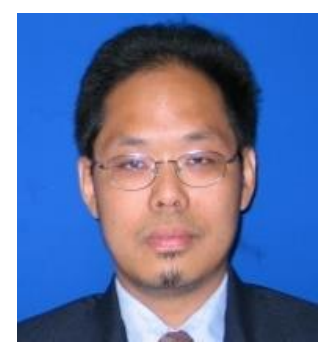

Mohammad Faiz Liew Abdullah received BSc (Hons) in Electrical Engineering (Communication) in 1997, Dip Education in 1999 and MEng by research in Optical Fiber Communication in 2000 from University of Technology Malaysia (UTM). He completed his PhD in August 2007 from The University of Warwick, United Kingdom in Wireless Optical Communication Engineering. He started his career as a lecturer at Polytechnic Seberang Prai (PSP) in 1999 and was transferred to UTHM in 2000 (formerly known as PLSP).

At present he is a senior lecturer in the Department of Communication Engineering, Faculty of Electrical \& Electronic Engineering, University Tun Hussein Onn Malaysia (UTHM). He had 10 years' experience of teaching in higher education, which involved the subject Optical Fiber Communication, Advanced Optical Communication, Advanced Digital Signal Processing and etc. His research area of interest are wireless and optical communication and robotic in communication.

How to cite this paper: M. F. Ghanim, M. F. L. Abdullah,"Analysis of MC-CDMA System in Mobile Communications", International Journal of Information Technology and Computer Science(IJITCS), vol.4, no.12, pp.87-94, 2012. DOI: $10.5815 /$ ijitcs.2012.12.10 